\title{
Questionnaire-based Study to Assess Knowledge of Preanalytical Phase of Laboratory Testing Among Trainee Doctors in a Tertiary Care Hospital Medical College
}

\author{
Kalpana Ketan Kulkarni ${ }^{1}$ Anup Pravin Bhandari ${ }^{1}$ Ashwin Keshavan Unni ${ }^{1}$
}

${ }^{1}$ Department of Pathology, BJ Govt. Medical College, Pune, Maharashtra, India

Address for correspondence Anup Pravin Bhandari, MBBS, MD, Department of Pathology, First floor, BJ Govt. Medical College, Jayprakash Narayan Road, Near Railway Station, Pune, Maharashtra, 411001, India (e-mail: anupbhandari154@gmail.com).

\begin{abstract}
Keywords

- Preanalytical error

- questionnaire

- automation

- order of draw

- coagulation testing

Objective To determine the knowledge base of common laboratory practices related to preanalytical phase of laboratory testing among medical interns and first-year postgraduate residents in a tertiary care hospital medical college.

Materials and methods Questionnaire-based survey on preanalytical phase of laboratory testing was conducted among 208 participants, who volunteered and were MBBS interns and first-year postgraduate residents in a medical college, over the period from June 2018 to December 2019. A total of 15-item, multiple-choice questions (MCQs) were included, of which four were opinion-based and not considered for analysis. Responses were further categorized based on percentages with correct response, so we could identify specific knowledge points which need training.

Statistical analysis Results were analyzed using Microsoft Excel functions and a simple calculator.

Results A total of 208 participants were included in the study, which consisted of an equal number of interns (104) and first-year postgraduate residents (104). The term "preanalytical error" was known to $62.5 \%$ of participants. Only $9.62 \%$ participants took formal training in phlebotomy. Topics related to questions like coagulation testing, ideal fasting duration, mixing of blood, and order of draw received less than $40 \%$ correct response which meant that it requires more training.

Conclusions In this era of evidence-based medicine, central laboratory plays a pivotal role in patient management, and quality of laboratory results are of paramount importance. Over a period of time, automation technology has reduced analytical phase errors to the minimum. Most errors reported are part of the preanalytical phase, and it has been found that a majority of them are committed due to a lack of knowledge and skills. In order to improve the preanalytical phase and make it as error-free as possible, the staff (technicians, nurses, and trainee doctors) should be constantly motivated and trained.
\end{abstract}

DOI https://doi.org/ 10.1055/s-0040-1720945 ISSN 0974-2727. (c) 2020. The Indian Association of Laboratory Physicians.

This is an open access article published by Thieme under the terms of the Creative Commons Attribution-NonDerivative-NonCommercial-License, permitting copying and reproduction so long as the original work is given appropriate credit. Contents may not be used for commercial purposes, or adapted, remixed, transformed or built upon. (https://creativecommons.org/licenses/by-nc-nd/4.0/)

Thieme Medical and Scientific Publishers Pvt. Ltd., A-12, 2nd Floor, Sector 2, Noida-201301 UP, India 


\section{Introduction}

Today, medical laboratories play a significant role in the health care system and the decision-making of clinical doctors with regard to their patients. According to official data, 60 to $70 \%$ of clinical decisions about hospitalization, discharge, and prescription are based on laboratory results. ${ }^{1}$ Clinical tests enjoy a high-status in screening, treatment follow-up, and assessment of response to treatment. Given this high-percentage and the significant role they play, the quality of laboratory tests is of high-importance. ${ }^{2}$ In other words, quality is the cornerstone of management in a laboratory, and it needs to conform to the highest standards. Laboratory services need to be accurate, precise, and quick to prove effective. ${ }^{3}$ Errors might happen throughout the process. These errors are categorized into three stages, depending on whether they are done before, during, or after analysis: preanalytic, analytic and postanalytic phase errors, respectively, ${ }^{4,5}$

Since there are no definite methods of sampling, most errors take place before the test and just before the sample is being prepared (it includes specimen collection, and handling and processing physiological variables and endogenous variables). Some of the preanalytical variables such as specimen collection can be controlled, while knowledge of uncontrollable variables needs to be well-understood, in order to be able to separate their effects from disease-related changes affecting the laboratory results. ${ }^{6}$

Preanalytical errors are reported to be up to $70 \%$ in various studies such as Plebani et $\mathrm{al}^{2}$ and Juli et al. ${ }^{7}$ In the recent past, with advancement in laboratory technology and automation, interphasing and digitalization of reports, human errors in analytical and postanalytical phases have been largely reduced. Since the quality is interconnected, precision and accuracy are not the only guarantors of the quality. From the very beginning, all three stages need to be under monitoring and quality control with precision and accuracy.

Most of the medical colleges in India bear tremendous patient load, and the majority of laboratory preanalytical work is performed by medical interns and residents. It has been observed that they lack formal training and adequate technical knowledge about the preanalytical phase, which leads to many erroneous laboratory test results.

The present questionnaire-based study was conducted with the objective to analyze the knowledge base of common laboratory practices among medical interns and residents in a tertiary medical college hospital. It will help us to plan training programs with lesser known topics, so as to improve laboratory test quality.

\section{Materials and Methods}

\section{Design of Study}

This was a cross-sectional observational study conducted after ethical committee clearance. The participants who volunteered were MBBS interns and first-year postgraduate residents in a medical college during the period from June 2018 to December 2019; all of them possessed a MBBS passing certificate.
A hard paper copy containing 15 -item, multiple-choice questionnaire (MCQ) with questions related to common laboratory practices was given to assess the knowledge base of 208 participants. Questions were prevalidated and approximately 10 minutes were given to respond. Time calculated for each question was approximately 45 seconds as most central MCQ examinations like NEET are prescribed. This MCQ consisted of the questions which focused on preanalytical variables like patient preparation (fasting and postprandial), sample collection, minimum amount of the sample required, various anticoagulants used in various analysis, etc.

Question numbers 1, 2, 3 and 5 were subjective and opinion-based, while the remaining questions were objective and analyzed for correct answer.

Responses were analyzed as percentages of correct answer and most commonly given answer. These responses were further categorized as less than $40 \%$ with correct answer, 40 to $80 \%$ with correct answer, and more than $80 \%$ with correct answer for our convenience. By making these categories, specific technical knowledge points were identified which could be stressed upon in designing training programs.

\section{Statistical Analysis}

All the data collected are entered in Microsoft Excel and analyzed in percentages using a simple calculator.

\section{Results}

A total of 208 students participated in this cross-sectional observational study. Of these, 104 were medical interns who had just passed their final MBBS examination, and 104 were PG residents who had taken admission for PG course in various specialties.

- Table 1 shows question-wise response of participants. Most of participants 190 (90.90\%) were happy with laboratory results (Question 1), with only $12(5.7 \%)$ saying faulty laboratory results are very frequent. Seven participants did not opine on the accuracy of laboratory results.

The term "preanalytical error" was known to 130 participants (62.5\%). A large number of participants (188, 90.38\%) said that they did not receive formal training in phlebotomy. Few commonly performed tests require fasting condition for, at least, specific durations, for example, lipid profile and blood sugar testing. Ideal fasting duration for lipid profile testing is 9 to 12 hours, while that for blood sugar is 6 to 8 hours. ${ }^{8}$ When participants were asked question about lipid profile, only 74 replied correctly (35\%), while most of them thought it is 6 to 8 hours, which is clearly inadequate fasting time for lipid profile.

When we asked about the type of bulb (in-house prepared or vacutainer) used for blood collection, 199 (95.69\%) participants knew about vacutainer use, while only 9 (4.31\%) did not respond.

For routine adult blood collection, needle with gauze number, 22 while for pediatric, small bore needle number 24 is preferred. About 97 (46.83\%) and 142 (68.27\%) participants responded with right answer for blood collection, respectively. 
Most interns and residents responded correctly (question 7-87.98\% and question 9-62.5\%) about amount of blood collected for routine testing in adult and pediatric patients, respectively.

Most respondents got it wrong (correct response-28.46\%) when asked about citrate bulb blood: anticoagulant ratio. Proper technique for mixing blood involves inversion technique for 8 to 10 times in a slow constant manner. ${ }^{9}$ Plain bulb must be kept steady and upright for serum separation. Many participants knew about plain bulb handling $(66.87 \%$ correct response), while only $37.01 \%$ participants correctly knew about the mixing technique of anticoagulant bulbs.
When asked about the accepted time interval for prothrombin time testing, only 48 (23.07\%) trainee doctors out of $208 \mathrm{knew}$ about the importance of time interval and accepted limits in coagulation testing.

A large number of participants (159/208-76.44\%) were unaware of the term "order of draw" and its importance. When asked about preference of site for blood collection in IPD patients, half of the trainee doctors (103/208-49.78\%) knew the preferred site is the opposite arm if IV catheter is inserted in one arm.

- Table 2 shows categorization of topics based on questions which received maximum right answers to minimum

Table 1 Frequency distribution of students according to responses given to questionnaire

\begin{tabular}{|c|c|c|c|c|}
\hline $\begin{array}{l}\text { Question } \\
\text { number }^{\mathrm{a}}\end{array}$ & Question (correct response) & $\begin{array}{l}\text { Correct } \\
\text { response (in \%) }\end{array}$ & $\begin{array}{l}\text { Most common } \\
\text { response } \\
\text { (in \%) (response) }\end{array}$ & $\begin{array}{l}\text { No } \\
\text { response (in\%) }\end{array}$ \\
\hline 1 & How often do you get faulty laboratory results? & \multicolumn{3}{|c|}{ (Frequent--5.7\%, few-90.90\%, do not know-3.4\%) } \\
\hline 2 & Are you aware about pre-analytical error? & \multicolumn{3}{|c|}{ (Yes-62.5\%, no-37.5\%) } \\
\hline 3 & Are you trained in phlebotomy? & \multicolumn{3}{|c|}{ (Yes-9.62\%, no-90.38\%) } \\
\hline 4 & $\begin{array}{l}\text { What is the ideal fasting duration for lipid profile } \\
\text { analysis? ( } 9-12 \text { hours) }\end{array}$ & 35.57 & 45.19 (6-8 hours) & 8 \\
\hline 5 & Which collection bulbs do you use? & \multicolumn{3}{|c|}{$\begin{array}{l}\text { (In-house prepared-0\%, vacutainer-95.69\%, do not } \\
\text { know-4.31\%) }\end{array}$} \\
\hline 6 & $\begin{array}{l}\text { Which needle size should be used for phlebotomy in } \\
\text { adults? }(22 \mathrm{~g})\end{array}$ & 46.83 & $N A^{b}$ & 17 \\
\hline 7 & $\begin{array}{l}\text { Ideally how much blood should be collected for a } \\
\text { hematological or biochemical analysis in adults? (5-7 } \\
\mathrm{mL} \text { ) }\end{array}$ & 87.98 & NA & 1 \\
\hline 8 & $\begin{array}{l}\text { Which needle size should be used for phlebotomy in } \\
\text { pediatric patients? }(24 \mathrm{~g})\end{array}$ & 68.27 & NA & 8 \\
\hline 9 & $\begin{array}{l}\text { How much blood is taken for laboratory diagnosis in } \\
\text { case of pediatric patients? ( } 2-5 \mathrm{ml})\end{array}$ & 62.5 & NA & 3 \\
\hline 10 & $\begin{array}{l}\text { What should be the anticoagulant: blood ratio in } \\
\text { citrate bulb? (1:9) }\end{array}$ & 28.36 & $\mathrm{NA}$ & 13 \\
\hline 11 & $\begin{array}{l}\text { What do you do after you collect blood in an EDTA } \\
\text { vacutainer tube? (Invert it around 8-10 times) }\end{array}$ & 37.01 & NA & 3 \\
\hline 12 & $\begin{array}{l}\text { What do you do after you collect blood in a plain } \\
\text { vacutainer tube? (Let it stand) }\end{array}$ & 66.82 & NA & 3 \\
\hline 13 & $\begin{array}{l}\text { Within how much time after blood collection should } \\
\text { the prothrombin time preferably be calculated for } \\
\text { accurate results? ( } 4 \text { hours) }\end{array}$ & 23.07 & 49.51 (3 hours) & 20 \\
\hline 14 & $\begin{array}{l}\text { In which bulb should the patients' first blood sample } \\
\text { be transported in? (citrate) }\end{array}$ & 23.55 & $\mathbf{5 2 . 8 8}$ (EDTA) & 6 \\
\hline 15 & $\begin{array}{l}\text { If the patient is on IV, then how do you collect his/her } \\
\text { blood? (any other vein from the other arm) }\end{array}$ & 49.52 & NA & 6 \\
\hline
\end{tabular}

Note: Bold values indicate questions where most common response is incorrect response.

auestion numbers 1, 2, 3 and 5 are opinion-based questions and are not considered for this analysis.

${ }^{b} \mathrm{NA}$ stands for not applicable when the most common response is the correct response.

Table 2 Categorization of question responses based on percentages with correct answer

\begin{tabular}{|c|c|c|c|}
\hline & \multicolumn{3}{|c|}{ Percentages of participants with correct response } \\
\hline & Less than $40 \%$ & 40 to $80 \%$ & More than $80 \%$ \\
\hline Question numbers & $4,10,13,14$ & $6,8,9,11,12,15$ & 7 \\
\hline $\begin{array}{l}\text { Topics related to } \\
\text { questions }\end{array}$ & $\begin{array}{l}\text { Fasting duration for lipid } \\
\text { profile analysis, } \\
\text { Coagulation testing, } \\
\text { Order of draw. }\end{array}$ & $\begin{array}{l}\text { Needle size for phlebotomy, } \\
\text { Adequacy of amount of blood required } \\
\text { for pediatric testing, } \\
\text { Selection of site for venepuncture. }\end{array}$ & $\begin{array}{l}\text { Adequacy of amount of blood } \\
\text { required for adult testing }\end{array}$ \\
\hline
\end{tabular}


right answers. Most participants gave wrong answers to question numbers $4,10,13$ and 14 (less than $40 \%$ correct answers), while only question number 7 received more than $80 \%$ correct response. Other questions received mix response (40-80\% correct response).

\section{Discussion}

As most of participants (90.90\%) were happy about laboratory results, it shows strong trust between clinician and laboratory people, which ultimately helps in fewer double checking of results and speedy management.

Awareness of preanalytical process plays a very important role in quality assurance of laboratory test results. In our study, although the term "preanalytical error" was known to 130 participants (62.5\%), efforts should be made to increase this number. Studies by Plebani et $\mathrm{al}^{2}$ and Da Rin ${ }^{10}$ showed preanalytical errors account for the major reason behind erroneous laboratory results. Specific training programs for interns, postgraduate residents and others who are chiefly involved in clinical sample collection should be performed periodically to reduce these errors. Also, instruction manuals, pictorial charts and guidelines about standard sample collection method and transport are to be made available in the wards and OPDs beside laboratory. This would help to maintain awareness.

Correct phlebotomy procedure is very crucial and the single most important step in the preanalytical process. Our study showed only $9.62 \%$ of participants received formal training in phlebotomy, thus lack of phlebotomy training is clearly an area that needs emphasis in the MBBS curriculum. The study by Lima-Oliveira et al supported that formal training improves quality of laboratory test results drastically. ${ }^{11}$

Few commonly performed tests require fasting condition for, at least, specific durations, for example, lipid profile and blood sugar testing. Ideal fasting duration for lipid profile testing is 9 to 12 hours, while that for blood sugar is 6 to 8 hours. ${ }^{8}$ Most of the participants (65\%) thought fasting duration for lipid profile testing is 6 to 8 hours which is clearly inadequate. This can lead to falsely high-test results and unnecessary drug management. We concurred that knowledge about fasting duration for common tests should be repeatedly told in the training induction program, so as to minimize falsely elevated test values.

Previously, our institute was preparing in-house bulbs for blood collection which resulted in many errors in laboratory results due to powder-based anticoagulation and the fact that the quality of anticoagulation was inconsistent, leading to high frequency of clotted samples. From the last few years, we have shifted to commercially available vacutainers which have reduced clotting of samples to a minimum. When we asked about this, 199 (95.69\%) participants knew about vacutainer use, while only 9 (4.31\%) failed to answer.

Selection of needle size is very important for blood collection, as it varies with age of patient, superficial vein anatomy, as well as quantity of blood required. For routine adult blood collection, needle with gauze number 22 , while for pediatric, small bore needle number 24 is preferred. About 97 (46.83\%) and $142(68.27 \%)$ participants responded with right answer for blood collection, respectively. Although there is no strict rule for needle use, choosing correct needle is very crucial as it avoids double pricks and inadequate sampling.

There is no standard fill quantity mentioned for various vacutainers, except for citrate bulbs, which have an indicator mark on the tube to maintain blood: anticoagulant ratio 9:1. The minimum quantity required for EDTA tubes is mentioned on the tubes, but the results did not vary significantly, even if half of the stated quantity was collected, according to a study by $\mathrm{Xu}$ et al. ${ }^{12}$ Most interns and residents responded correctly (question $7-87.98 \%$ and question 9-62.5\%) about amount of blood collected for routine testing in adult and pediatric patients, respectively. Specifically designed pediatric vacationers are now available, which are adjusted for low-volume fill. Most respondents got it wrong (correct response-28.46\%) when asked about citrate bulb blood: anticoagulant ratio. This fact leads us to believe that knowledge about coagulation testing and its requirement is very poor among doctors and trainees, and it needs special emphasis.

It is routinely observed that hemolyzed samples received are unsuitable for testing and attributed to poor handling and mixing techniques. Rough shaking of bulbs for mixing of blood can lead to hemolysis. Proper technique for mixing blood involves inversion technique for 8 to 10 times in a slow constant manner. ${ }^{12}$ Plain bulb must be kept steady and upright for serum separation. Many participants knew about plain bulb handling (66.87\% correct response), while only $37.01 \%$ participants correctly knew about the mixing technique. Others followed unscientific methods, unaware of its consequences on laboratory results. Pictorial charts are the best method of improving awareness of this technique, which we decided to use in our training programs. These can be used at collection sites and wards for staff information.

Due to huge workload in medical college hospitals, most of the residents and intern doctors tend to send samples in bulk from wards at their convenience after emergency work is over. Many a times this leads to variable time interval for laboratory blood sample collection and processing. Coagulation testing results are greatly affected by variation in storage temperature and time interval unlike other routine tests up to now; the Clinical Laboratory and Standards Institute (CLSI) Guideline H21-A56 recommends that most coagulation parameters must be evaluated within 4 hours, except tests aimed at monitoring treatments with full-dose unfractionated heparin for whom the delay must not exceed 2 hours. ${ }^{13}$ When asked about accepted time interval, only 48 (23.07\%) trainee doctors out of 208 knew about the importance of time interval and accepted limits in coagulation testing.

Often more than one tube is required for different blood tests. These blood tubes may contain different additives which may affect certain results should they contaminate another tube. For this reason, an order of draw was established. ${ }^{14}$ 
An opinion paper by Cornes et $\mathrm{al}^{14}$ in European Federation of Clinical Chemistry and Laboratory Medicine (EFLM) stated that order of draw should start with blood culture tube (color varies), followed by citrate tube (light blue), clot activator (red), heparin (green), EDTA (lavender), fluoride (gray) in that order. Large number of participants (159/208-- 76.44\%) were unaware of this order of draw.

WHO guidelines on best practices in phlebotomy mentioned few commonly occurring errors in patient with IV catheters. ${ }^{15}$ While IV lines provide a means of direct vascular access for infusion fluids, collection of specimens through these lines can result in contamination of the specimen with the contents of the line. Whenever possible, specimens should be collected from the arm opposite the line to avoid contamination. Specimens should not be collected distal to a catheter because fluids tend to pool in the periphery of the limb. Collection of samples proximal to a catheter will be diluted by the infusion fluid. ${ }^{15}$

When vascular access is limited, a specimen may need to be collected from an IV line. This decision should only be made after weighing the risk of specimen contamination versus the risk of phlebotomy from another site. Before drawing a specimen from a line, the infusion fluid should be completely stopped for several minutes and an amount of blood equal to three or more times the dead space of the catheter should be discarded. ${ }^{15}$

When asked about preference of site for blood collection in IPD patients, nearly half of trainee doctors (103/208-49.78\%) knew the preferred site is the opposite arm if IV catheter is inserted in one arm. As contamination or dilution of specimen can seriously affect laboratory test results, this error must be minimized by inclusion of these guidelines in every phlebotomy training program.

- Table 2 summarizes results of all questions according to percentages of correct responses. Questions which received least correct response (less than 40\%) were related to topics of coagulation testing, ideal fasting duration for lipid profile, and order of draw. The reason for this might be because few topics such as coagulation testing are complex, while others like variable fasting duration for sugar and lipids are subtle to understand. Order of draw is very new concept and vastly unknown to new interns and first-year postgraduate residents. These topics need special emphasis in the training program.

\section{Limitation of Study}

Preanalytical phase of laboratory test is strongly associated with proper knowledge as well as the skills of a person. However, our study did not take into consideration the "skill" part of preanalytical process and emphasized only on the knowledge part.

\section{Conclusion}

Preanalytical phase of laboratory testing plays a crucial role in quality assurance of test results, as majority of errors occur in this phase. Although most participants are aware of the term "preanalytical error," only $9.62 \%$ received informal training for phlebotomy. So, it is necessary to increase awareness and introduce formal training in the MBBS curriculum.

Use of vacutainers, selection of proper needle size for pediatric and adult patients, and adequate sample volume are well-understood topics among interns and residents.

Topics like coagulation testing, ideal fasting duration, and order of draw are very poorly known among interns and first-year postgraduate residents (less than 40\% responded correctly to questions). These topics need special emphasis in the training program.

\section{Financial Disclosure}

None declared.

\section{Details of Earlier Presentation}

None.

\section{Author's role/participation in the authorship of the manuscript}

First author: concept and manuscript preparation. Second author: Conducting study and result analysis, preparing manuscript, and carrying out correspondence. Third author: Conducting study and compiling result.

\section{Conflicts of Interest}

Dr. Bhandari reports grants from the Indian Council of Medical Research (ICMR) under short-term studentship (STS) outside the submitted work. Dr. Kulkarni reports grants from ICMR under STS outside the submitted work. Dr. Unni reports grants from ICMR under STS outside the submitted work.

\section{References}

1 Carraro P, Plebani M. Errors in a stat laboratory: types and frequencies 10 years later. Clin Chem 2007;53(7):1338-1342

2 Plebani M, Carraro P. Mistakes in a stat laboratory: types and frequency. Clin Chem 1997;43(8 Pt 1) :1348-1351

3 Hooijberg E, Leidinger E, Freeman KP. An error management system in a veterinary clinical laboratory. J Vet Diagn Invest 2012;24(3):458-468

4 Aakre KM, Langlois MR, Watine J, et al. Critical review of laboratory investigations in clinical practice guidelines: proposals for the description of investigation. Clin Chem Lab Med 2013;51(6):1217-1226

5 Chhillar N, Khurana S, Agarwal R, Singh NK. Effect of pre-analytical errors on quality of laboratory medicine at a neuropsychiatry institute in north India. Indian J Clin Biochem 2011;26(1):46-49

6 Romero A, Cobos A, GómezJ, Muñoz M. Role of training activities for the reduction of pre-analytical errors in laboratory samples from primary care. Clin Chim Acta 2012;413(1-2):166-169

7 Julie A. Hammerling, A review of medical errors in laboratory diagnostics and where we are today. Lab Med 2012;43(2): 41-44

8 Janovsky CC, Laurinavicius A, Cesena F, et al. Impact of self-reported fasting duration on lipid profile variability, cardiovascular risk stratification and metabolic syndrome diagnosis. Arch Endocrinol Metab 2018;62(2):187-192

9 Lippi G, Salvagno G, Montagnana M, Banfi G, Guidi G. Evaluation of different mixing procedures for K2 EDTA primary samples on hematological testing. Labmedicine. 2007;38:723-725 
10 Da Rin G. Pre-analytical workstations: a tool for reducing laboratory errors. Clin Chim Acta 2009;404(1):68-74

11 Lima-Oliveira G, Lippi G, Salvagno GL, Montagnana M, Picheth G, Guidi GC. Impact of the phlebotomy training based on CLSI/NCCLS H03-a6 - procedures for the collection of diagnostic blood specimens by venipuncture. Biochem Med (Zagreb)2012; 22(3):342-351

12 Xu M, Robbe VA, Jack RM, Rutledge JC. Under-filled blood collection tubes containing K2EDTA as anticoagulant are acceptable for automated complete blood counts, white blood cell differential, and reticulocyte count. Int J Lab Hematol 2010; 32(5):491-497

13 Toulon P, Metge S, Hangard M, Zwahlen S, Piaulenne S, Besson V. Impact of different storage times at room temperature of unspun citrated blood samples on routine coagulation tests results. Results of a bicenter study and review of the literature. Int J Lab Hematol 2017;39(5):458-468
14 Cornes M, van Dongen-Lases E, Grankvist K, et al; Working Group for Preanalytical Phase (WG-PRE), European Federation of Clinical Chemistry and Laboratory Medicine (EFLM). Order of blood draw: Opinion Paper by the European Federation for Clinical Chemistry and Laboratory Medicine (EFLM) Working Group for the Preanalytical Phase (WG-PRE). Clin Chem Lab Med 2017;55(1):27-31

15 WHO. WHO guidelines on drawing blood best practices in phlebotomy. Available at: http://www.euro.who.int/_data/ assets/pdf_file/0005/268790/WHO-guidelines-on-drawingblood-best-practices-in-phlebotomy-Eng.pdf?ua-1. Accessed July 9, 2020 